\title{
ON THE HOLOMORPHICITY OF HARMONIC MAPS FROM COMPACT KÄHLER MANIFOLDS TO HYPERBOLIC RIEMANN SURFACES
}

\author{
KAORU ONO
}

(Communicated by Haynes R. Miller)

\begin{abstract}
A BSTRACT. We give a sufficient condition in order that a harmonic map from a compact Kähler manifold with negative first Chern class to a compact hyperbolic Riemann surface be \pm holomorphic. The above condition generalizes that of Eells and Wood concerning harmonic maps between Riemann surfaces. As a corollary we get a generalization of Kneser's theorem.
\end{abstract}

1. Introduction. It is an interesting problem to find out the condition which implies the holomorphicity of harmonic maps between Kähler manifolds. In fact, Lichnerowicz has proved that holomorphic maps between Kähler manifolds are harmonic with respect to the Kähler metrics [6]. Conversely the following facts are known.

(A) (Eells and Wood [4]) Let $X$ and $Y$ be compact Riemann surfaces and $f$ a harmonic map from $X$ to $Y$ with respect to some Kähler metrics. If $f$ satisfies the following condition then $f$ is \pm holomorphic (i.e. holomorphic or anti-holomorphic):

$$
e(X)+|\operatorname{deg}(f) \cdot e(Y)|>0
$$

where $e(X)$ and $e(Y)$ are the Euler numbers of $X$ and $Y$ respectively and $\operatorname{deg}(f)$ is the degree of the map $f: X \rightarrow Y$.

(B) (Siu [7]) Let $M$ and $N$ be compact Kähler manifolds and assume that $N$ has strongly negative curvature in the sense of Siu. Let $f$ be a harmonic map from $M$ to $N$ with respect to the Kähler metrics. If there is a point in $M$ where the rank of $d f$ is greater than or equal to four, then $f$ is \pm holomorphic.

In this paper, we shall prove the following

THEOREM 1. Let $M$ be an m-dimensional compact Kähler manifold with negative first Chern class, $N$ a compact hyperbolic Riemann surface (i.e. closed Riemann surface with the metric of constant negative curvature), and $f$ a harmonic map from $M$ to $N$. If $f$ satisfies

$$
m \cdot\left|f^{*} c_{1}(N) \cdot c_{1}(M)^{m-1}[M]\right|>\left|c_{1}(M)^{m}[M]\right|,
$$

then $f$ is \pm holomorphic.

This may be considered as a generalization of the above result (A) of Eells and Wood. Result (B) of Siu cannot be applied directly to the above case, but a lemma in [8] and [9] is used in the course of proof of Theorem 1.

Received by the editors November 6, 1986.

1980 Mathematics Subject Classification (1985 Revision). Primary 58E20; Secondary 53C55.

Key words and phrases. Harmonic map, holomorphic map, Kähler manifold, Einstein-Hermitian vector bundle, semistable vector bundle.

(C) 1988 American Mathematical Society $0002-9939 / 88 \$ 1.00+\$ .25$ per page 
As a corollary of Theorem 1, we can obtain the following

THEOREM 2. Let $M$ and $N$ be as in Theorem 1. For any continuous map $f$ from $M$ to $N$, we get the inequality

$$
\left|f^{*} c_{1}(N) \cdot c_{1}(M)^{m-1}[M]\right| \leq m \cdot\left|c_{1}(M)^{m}[M]\right| .
$$

This may be considered as a generalization of Kneser's theorem [4].

The author wishes to express deep appreciation to Professor Akio Hattori for his advice and continuous encouragement.

2. Review of holomorphic vector bundles. In this section, we recall the definitions and fundamental properties of semistable vector bundles and EinsteinHermitian vector bundles. The details can be found in [5].

Let $M$ be an $m$-dimensional compact Kähler manifold and $\Phi$ the Kähler form on $M$.

DEFINITION (2.1). A holomorphic vector bundle $E$ over $M$ is called a $\Phi$ semistable vector bundle if and only if for any coherent subsheaf $S$ satisfying $0<$ rank $S<\operatorname{rank} E$, the following condition is satisfied:

$$
\int_{M} \frac{c_{1}(S)}{\operatorname{rank} S} \wedge \Phi^{m-1} \leq \int_{M} \frac{c_{1}(E)}{\operatorname{rank} E} \wedge \Phi^{m-1} .
$$

We call $(E, h, \nabla)$ a Hermitian vector bundle if $E$ is a holomorphic vector bundle over $M$ with a Hermitian metric $h$ and a Hermitian connection $\nabla$.

DEFINITION (2.2). A Hermitian vector bundle $(E, h, \nabla)$ over $M$ is called an Einstein-Hermitian vector bundle if and only if $K=\phi \cdot I_{E}$ where $K$ is the Ricci curvature of $(E, h, \nabla)$ (i.e. the trace of the curvature with respect to the Kähler metric on $M), \phi$ is a function on $M$, and $I_{E}$ is the identity bundle isomorphism of $E$.

We shall use the following lemma in the proof of Theorem 1.

LEMMA (2.3). (1) Every holomorphic line bundle with any Hermitian metric becomes an Einstein-Hermitian vector bundle.

(2) The tensor product of any two Einstein-Hermitian vector bundles is also an Einstein-Hermitian vector bundle.

(3) The dual bundle of an Einstein-Hermitian vector bundle is also an EinsteinHermitian vector bundle.

(4) Every Einstein-Hermitian vector bundle is a $\Phi$-semistable vector bundle.

(5) Let $(M, \Phi)$ be an Einstein-Kähler manifold. Then the tangent bundle TM of $M$ with the Einstein-Kähler metric is an Einstein-Hermitian vector bundle on $(M, \Phi)$.

REMARK (2.4). If $M$ is a compact Kähler manifold with negative first Chern class, then there is a unique Einstein-Kähler metric on $M$ up to a constant multiple [10]. By Lemma (2.3)(5) the tangent bundle $T M$ of $M$ is $\Phi$-semistable where $\Phi$ is the Kähler form of the Einstein-Kähler metric.

3. Proof of Theorem 1. Let $M$ and $N$ be Kähler manifolds, and $f$ a smooth map from $M$ to $N$. We decompose the complexified differential $d^{\mathrm{C}} f: T M \otimes_{\mathbf{R}} \mathrm{C} \rightarrow$ $T N \otimes_{\mathbf{R}} C$ of $f$ as follows:

$$
\begin{array}{ll}
\partial f: T^{\prime} M \rightarrow T^{\prime} N, & \bar{\partial} f: T^{\prime \prime} M \rightarrow T^{\prime} N, \\
\partial \bar{f}: T^{\prime} M \rightarrow T^{\prime \prime} N, & \overline{\partial f}: T^{\prime \prime} M \rightarrow T^{\prime \prime} N,
\end{array}
$$


where $T^{\prime} M$ and $T^{\prime} N$ are the holomorphic tangent bundles of $M$ and $N$ respectively, and $T^{\prime \prime} M$ and $T^{\prime \prime} N$ are the antiholomorphic tangent bundles of $M$ and $N$ respectively.

We denote by $O_{M}$ the sheaf of holomorphic functions on $M$, and by $O_{M}(E)$ the sheaf of holomorphic cross sections of the holomorphic vector bundle $E$ over $M$.

We recall the following fact due to $\operatorname{Siu}[\mathbf{8}, \mathbf{9}]$.

LEMMA (3.1). Let $M$ be a compct Kähler manifold, $N$ a hyperbolic Riemann surface, and $f$ a harmonic map from $M$ to $N$ with respect to the Kähler metrics. Then the pullback of the tangent bundle of $N$ by $f$ becomes a holomorphic line bundle, and moreover, $\partial f$ is a holomorphic cross section of the tensor product bundle $T^{*} M \otimes f^{*} T N$, where we identify $T M$ and $T N$ with $T^{\prime} M$ and $T^{\prime} N$ respectively.

PROOF OF THEOREM 1. By Lemma (3.1), $\partial f$ is a holomorphic cross section of the bundle $T^{*} M \otimes f^{*} T N$. If $f$ is not antiholomorphic, then $\partial f$ is not identically zero on any subset of $M$. Therefore the $O_{M}$-subsheaf $\tau$ of $O_{M}\left(T^{*} M \otimes f^{*} T N\right)$ generated by $\partial f$ is isomorphic to $O_{M}$, so in particular $\tau$ is a coherent subsheaf of $O_{M}\left(T^{*} M \otimes f^{*} T N\right)$. By Remark (2.4) and Lemma (2.3)(3), $T^{*} M$ is an EinsteinHermitian vector bundle with respect to the Einstein-Kähler metric on $M$. On the other hand, since $f^{*} T N$ is a holomorphic line bundle, it is an Einstein-Hermitian vector bundle with respect to any Kähler metric on $M$ by Lemma (2.3)(1). By Lemma (2.3)(2) and (4), $T^{*} M \otimes f^{*} T N$ is a $\Phi$-semistable vector bundle where $\Phi$ is the Kähler form of the Einstein-Kähler metric. Applying the condition in the definition of $\Phi$-semistable vector bundle to $\tau \subset O_{M}\left(T^{*} M \otimes f^{*} T N\right)$, we obtain

$$
0 \leq\left\{m \cdot f^{*} c_{1}(N)-c_{1}(M)\right\} \cdot\left\{-c_{1}(M)\right\}^{m-1}[M]
$$

since $c_{1}\left(T^{*} M \otimes f^{*} T N\right)=m \cdot f^{*} c_{1}(N)-c_{1}(M)$ and $\Phi$ represents the cohomology class $-c_{1}(M)$.

Now we consider $\bar{N}$ in place of $N$, where $\bar{N}$ is the Riemann surface with opposite complex structure on the underlying manifold of $N$. Similarly, if $f$ is not a holomorphic map from $M$ to $N$, we obtain

$$
\begin{aligned}
0 & \leq\left\{m \cdot f^{*} c_{1}(\bar{N})-c_{1}(M)\right\} \cdot\left\{-c_{1}(M)\right\}^{m-1}[M] \\
& =\left\{-m \cdot f^{*} c_{1}(N)-c_{1}(M)\right\} \cdot\left\{-c_{1}(M)\right\}^{m-1}[M] .
\end{aligned}
$$

From (3.2) and (3.3), we obtain

$$
m \cdot\left|f^{*} c_{1}(N) \cdot c_{1}(M)^{m-1}[M]\right| \leq\left|c_{1}(M)^{m}[M]\right| .
$$

On the other hand, by the assumption we have

$$
m \cdot\left|f^{*} c_{1}(N) \cdot c_{1}(M)^{m-1}[M]\right|>\left|c_{1}(M)^{m}[M]\right|
$$

which is a contradiction. Therefore $f$ is \pm holomorphic.

REMARK (3.4). We can give a slightly different, but essentially the same, proof of (3.2). For any Einstein-Hermitian vector bundle $(E, h, \nabla)$, there exists a function $\rho$ on $M$ such that $(E, \rho \cdot h, \nabla)$ has Ricci curvature $K$ satisfying the following condition [5]:

$$
K=c \cdot(\rho \cdot h), \quad \text { where } c=\frac{2 m \rho \cdot \int_{M} c_{1}(E) \cdot \Phi^{m-1}}{\int_{M} \Phi^{m}} .
$$


In our case $E=T^{*} M \otimes f^{*} T N$ and $\Phi$ represents the cohomology class $-c_{1}(M)$, therefore

$$
c=\frac{2 m \pi}{\left\{-c_{1}(M)\right\}^{m}[M]} \cdot\left\{m \cdot f^{*} c_{1}(N)-c_{1}(M)\right\} \cdot\left\{-c_{1}(M)\right\}^{m-1}[M] .
$$

Proposition 3 in [1] asserts that if $E$ has a nonzero holomorphic cross section then $c>0$. By the assumption, $T^{*} M \otimes f^{*} T N$ has a nonzero holomorphic cross section $\partial f$, hence we obtain (3.2).

4. The upper bound of the energy functional of holomorphic maps. The energy of the smooth map $f$ is defined as

$$
E(f)=\frac{1}{2} \int_{M}\|d f\|^{2} d v_{M}
$$

where the norm $\|d f\|$ is defined by the Kähler metrics on $M$ and $N$, and $d v_{M}$ is the Riemannian volume form on $M$. We shall prove the following

PROPOSITION (4.1). Let $M$ be a Kähler manifold with the Ricci curvature bounded from below by $k / \operatorname{dim}_{\mathbf{R}} M$ for some negative constant $k, N$ a Kähler manifold with nonpositive sectional curvature and holomorphic sectional curvature bounded from above by some negative constant $K$, and $f$ a holomorphic map from $M$ to $N$. Then $f$ satisfies the inequality

$$
E(f) \leq \frac{|k| \cdot \operatorname{vol}(M)}{2|K|} .
$$

PROOF. The well-known Bochner-Weitzenböck formula for harmonic maps (cf. $[2,3])$ states

$$
\begin{aligned}
\frac{1}{2} \Delta|d f|^{2}= & -|\nabla d f|^{2}+\sum_{s, t}\left\langle R^{N}\left(d f\left(e_{s}\right), d f\left(e_{t}\right)\right) d f\left(e_{t}\right), d f\left(e_{s}\right)\right\rangle \\
& -\sum_{s}\left\langle\left(\operatorname{Ric}^{M}\left(e_{s}\right), d f\left(e_{s}\right)\right\rangle,\right.
\end{aligned}
$$

where $\left\{e_{s}\right\}$ is an orthonormal basis of $T M, R^{N}$ is the Riemannian curvature tensor of $N$ (we adopt the usual convention for curvature tensor instead of Milnor's one, so the above formula differs from the one in [2]), and $\mathrm{Ric}^{M}$ is the Ricci curvature tensor of $M$. We integrate the above formula on $M$ and obtain

$$
\begin{aligned}
0 \leq \int_{M}\left\{\sum _ { s , t } \left\langleR^{N}\left(d f\left(e_{s}\right), d f\left(e_{t}\right)\right) d f\left(e_{t}\right)\right.\right. & \left., d f\left(e_{s}\right)\right\rangle \\
& -\sum_{s}\left\langle d f\left(\operatorname{Ric}^{M}\left(e_{s}\right), d f\left(e_{s}\right)\right\rangle\right\} d v_{M} .
\end{aligned}
$$

In our case, we can choose an orthonormal basis $\left\{e_{i}\right\}$ satisfying $e_{2 i}=J e_{2 i-1}$, where $J$ is the complex structure on $M$. From the definition of the holomorphic sectional curvature, and the assumption of the proposition, we obtain

$$
\begin{aligned}
& \left\langle R^{N}\left(d f\left(e_{2 i-1}\right), d f\left(e_{2 i}\right)\right) d f\left(e_{2 i}\right), d f\left(e_{2 i-1}\right)\right\rangle \leq K\left\|d f\left(e_{2 i-1}\right)\right\|^{4} \\
& \left\langle R^{N}\left(d f\left(e_{2 i}\right), d f\left(e_{2 i-1}\right)\right) d f\left(e_{2 i-1}\right), d f\left(e_{2 i}\right)\right\rangle \leq K\left\|d f\left(e_{2 i}\right)\right\|^{4} .
\end{aligned}
$$


By the assumption of the proposition, we have

$$
\left\langle R^{N}\left(d f\left(e_{s}\right), d f\left(e_{t}\right)\right) d f\left(e_{t}\right), d f\left(e_{s}\right)\right\rangle \leq 0 .
$$

From (4.3) and (4.4), we derive

$$
\begin{aligned}
& \sum_{s, t}\left\langle R^{N}\left(d f\left(e_{s}\right), d f\left(e_{t}\right)\right) d f\left(e_{t}\right), d f\left(e_{s}\right)\right\rangle \\
& \quad \leq \sum_{s} K\left\|d f\left(e_{s}\right)\right\|^{4} \leq \frac{K}{\operatorname{dim}_{\mathbf{R}} M}\|d f\|^{4} .
\end{aligned}
$$

By the assumption of the proposition, we have

$$
\sum_{s}\left\langle d f\left(\operatorname{Ric}^{M}\left(e_{s}\right)\right), d f\left(e_{s}\right)\right\rangle \geq \frac{k}{\operatorname{dim}_{\mathbf{R}} M}\|d f\|^{2} .
$$

From (4.2), (4.5) and (4.6), we obtain

$$
0 \leq \int_{M}\left(|k| \cdot\|d f\|^{2}-|K| \cdot\|d f\|^{4}\right) d v_{M}
$$

Hence,

$$
\begin{aligned}
|k| \cdot \int_{M}\|d f\|^{2} d v_{M} & \geq|K| \cdot \int_{M}\|d f\|^{4} d v_{M} \\
& \geq \frac{|K|}{\operatorname{vol}(M)}\left(\int_{M}\|d f\|^{2} d v_{M}\right)^{2} .
\end{aligned}
$$

(The second inequality is obtained by the use of the Schwarz inequality.)

Thus we have proved $E(f) \leq|k| \cdot \operatorname{vol}(M) / 2|K|$.

5. Proof of Theorem 2. If $f$ satisfies

$$
m \cdot\left|f^{*} c_{1}(N) \cdot c_{1}(M)^{m-1}[M]\right| \leq\left|c_{1}(M)^{m}[M]\right|,
$$

then there is nothing to prove. So we assume that $f$ satisfies

$$
m \cdot\left|f^{*} c_{1}(N) \cdot c_{1}(M)^{m-1}[M]\right|>\left|c_{1}(M)^{m}[M]\right| .
$$

From a well-known theorem of Eells and Sampson [3], there is a harmonic map in any homotopy class of maps from $M$ to $N$. Therefore we may assume that $f$ is a harmonic map. We apply Theorem 1 to the above map $f$, showing that $f$ is \pm holomorphic. Without loss of generality, we can assume that $f$ is holomorphic. Then from Proposition (4.1), we obtain

$$
E(f) \leq \frac{|k| \cdot \operatorname{vol}(M)}{2 \cdot|K|}
$$

If we choose a Kähler metric on $N$ with constant sectional curvature equal to -1 , then the Kähler form $\omega_{N}$ represents $-c_{1}(N)$, where we adopt the following definition of the Kähler form:

$$
\omega=\frac{\sqrt{-1}}{2 \pi} \cdot g_{\lambda \bar{\mu}} d x^{\lambda} \wedge d \bar{z}^{\mu}, \quad \text { where } g_{\lambda \bar{\mu}} \text { is the Kähler metric tensor. }
$$

Now, it is easy to see that

$$
\frac{1}{m !} \cdot\left|f^{*} c_{1}(N) c_{1}(M)^{m-1}[M]\right|=\frac{1}{(2 \pi)^{m}} E(f),
$$


where the Kähler metric on $M$ is chosen as the one whose Kähler form represents $-c_{1}(M)$. The Ricci curvature of $M$ is equal to -1 times the metric tensor, and the volume of $M$ is equal to $(2 \pi)^{m} / m ! \cdot\left|c_{1}(M)^{m}[M]\right|$. Hence we obtain

$$
\left|f^{*} c_{1}(N) c_{1}(M)^{m-1}[M]\right| \leq m \cdot\left|c_{1}(M)^{m}[M]\right| \text {. }
$$

\section{BIBLIOGRAPHY}

1. S. K. Donaldson, Anti self-dual Yang-Mills connections over complex algebraic surfaces and stable vector bundles, Proc. London Math. Soc. (3) 50 (1985), 1-26.

2. J. Eells and L. Lemaire, Selected topics in harmonic maps, CBMS Regional Conf. Ser. in Math., Amer. Math. Soc., Providence, R. I., 1983.

3. J. Eells and J. H. Sampson, Harmonic mappings of Riemannian manifolds, Amer. J. Math. 86 (1964), 109-160.

4. J. Eells and J. C. Wood, Restrictions on Harmonic maps of surfaces, Topology 15 (1976), 263-266.

5. S. Kobayashi, Stable vector bundles and curvature, Lecture Notes from University of Tokyo, 1984.

6. A. Lichnerowicz, Applications harmoniques et varieétés Kählériennes, Sympos. Math. III (Bologna), 1970, pp. 341-402.

7. Y. T. Siu, The complex analyticity of harmonic maps and the strong rigidity of compact Kähler manifolds, Ann. of Math. (2) 112 (1980), 73-111.

8. _ - Some recent results in complex manifold theory related to vanishing theorems for semipositive case, Lecture Notes in Math., vol. 1111, Springer-Verlag, 1985, pp. 169-192.

9. __ Strong rigidity for Kähler manifolds and the construction of bounded holomorphic functions, Discrete Groups in Geometry and Analysis, Papers in Honor of G. D. Mostow on His Sixtieth Birthday (R. Howe, ed.), Birkhauser, Boston, Mass., 1987, pp. 124-151.

10. S. T. Yau, Calabi's conjecture and some new results in algebraic geometry, Proc. Nat. Acad. Sci. U.S.A. 74 (1977), 1798-1799.

Department of Mathematics, Faculty of Science, University of Tokyo, HONGO, TOKYO, 113 JAPAN 